\title{
SCRUB TYPHUS INFECTION AMONG PUO CASES IN THE PEDIATRIC POPULATION IN A TERTIARY CARE HOSPITAL
}

\author{
ANUPRIYA A ${ }^{1}$, RAJKUMAR B ${ }^{1}$, PRABHUSARAN N ${ }^{1 *}$, PRIYA BANTHAVI ${ }^{2}$ \\ ${ }^{1}$ Department of Microbiology, , Trichy SRM Medical College Hospital and Research Centre, Tiruchirappalli, Tamil Nadu, India. ${ }^{2}$ Department \\ of Pathology, Trichy SRM Medical College Hospital and Research Centre, Tiruchirappalli, Tamil Nadu, India. Email: leptoprabhu@gmail.com
}

Received: 18 February 2021, Revised and Accepted: 15 March 2021

\section{ABSTRACT}

Objectives: Scrub typhus is an acute, febrile, exanthematous illness. This disease is underdiagnosed in India due to its non-specific and varied clinical presentation, low index of suspicion among clinicians, and lack of diagnostic facilities. This study was carried out to know the seroprevalence of scrub typhus in children with PUO and to compare a rapid test with IgM enzyme-linked immunosorbent assay (ELISA) for the diagnosis of scrub typhus.

Methods: This cross-sectional analytical study was conducted for a period of 1 year. The study population comprised mainly 280 young children attending pediatric $\mathrm{OP}$ and in patients admitted to a tertiary care teaching hospital with fever and related symptoms. The serum samples were tested for Weil-Felix reaction, IgM ELISA, and rapid card test.

Results: The mean age group of the study population was 7-9 years, of which 20 cases were positive. The major predisposing factor for scrub typhus infection was vegetation around houses. The sensitivity and specificity of both, card test and IgM ELISA, were $100 \%$.

Conclusion: In this study, 7.1\% of febrile children were positive for scrub typhus. Leptospirosis, dengue, and typhoid were the common coinfections found in scrub typhus, positive children. Early identification of cases and treatment at the earliest will prevent complications.

Keywords: Febrile children, Orientia tsutsugamushi, Scrub typhus.

(C) 2021 The Authors. Published by Innovare Academic Sciences Pvt Ltd. This is an open access article under the CC BY license (http://creativecommons.org/ licenses/by/4.0/) DOI: http://dx.doi.org/10.22159/ajpcr.2021v14i5.41143. Journal homepage: https://innovareacademics.in/journals/index.php/ajpcr

\section{INTRODUCTION}

All over the world, fever in children is one of the most common reasons for parents to seek medical care. It remains among the most challenging clinical situations in diagnosis for health-care providers to approach and often involves referral to subspecialists. In Southeast Asia, scrub typhus infection is one important cause of acute undifferentiated fever. This mite borne illness is caused by the bacterium belonging to Rickettsia family, Orientia tsutsugamushi [1]. This infection is grossly underdiagnosed in India because of its non-specific clinical presentation, low index of suspicion among clinicians, limited awareness about the disease, and lack of diagnostic facilities [2]. Although the disease starts with non-specific symptoms, it may lead to high mortality (35-60\%) if diagnosis or appropriate therapy is delayed [3].

The majority of studies on rickettsial infections have been done in adult populations and there is a paucity of community-based data of its incidence and clinical profile in children despite epidemiological mention of children constituting up to half of scrub typhus cases in some regions [4]. Children once infected develop symptoms after an incubation period of 7-21 days. The clinical spectrum ranges from fever accompanied by nausea, vomiting, headache, and myalgia to fatal complications such as meningoencephalitis and even acute respiratory distress syndrome [5]. The delay in therapy in the Indian subcontinent is attributed to the lack of recognition or absence of the Eschar, a characteristic skin lesion that starts as a vesicular lesion at the site of the mite's bite.

The possible explanation for the absence of an Eschar in the Southeast population is said to be due to the variation in cutaneous immunity [6]. However, a study in South India among children with scrub typhus demonstrated the presence of Eschar in $46 \%$ of cases [7]. Following clinical suspicion, several tests are available for diagnosing scrub typhus, such as Weil-Felix test, IgM enzyme-linked immunosorbent assay (ELISA), and rapid test (immunochromatography). Out of which
Weil-Felix test, though cheap and easily available, is unreliable due to sharing of antigens while the rapid test is both economical and a "point of care" test. IgM ELISA testing has also been evaluated and found to be satisfactory in diagnosis of scrub typhus.

This study was carried out to determine the seroprevalence of scrub typhus in clinically suspected children and to compare the efficacy of rapid test with that of IgM ELISA in the diagnosis of scrub typhus.

\section{METHODS}

The present cross-sectional analytical study was conducted from the period of May 2019 to April 2020 on all children with acute undifferentiated febrile illness attending pediatric OP and in children admitted to a tertiary care teaching hospital. Universal sampling was done and all children presenting with fever and related symptoms were included in the study. There were a total of 280 children presenting with fever and related symptoms. The sample size was calculated from pre-existing studies in the literature with a prevalence rate of $23 \%$ in adults in the Pondicherry [7]. This study was approved and certified by the Institutional Ethics Committee (Ref: No. 636/TSRMMCH\&RC/ME1/2019-IEC No: 006 dated July 17, 2019) and written informed consent was obtained from all the participants included in this study.

\section{Inclusion criteria}

All children presenting with acute febrile illness due to infectious etiology were included in the study.

\section{Exclusion criteria}

Children presenting with fever due to metabolic causes or other noninfectious etiology were excluded from the study.

A structured pro forma was utilized for the current study. The details such as age, place where the child is residing, socioeconomic status, educational qualification of mother, source of drinking water, history 
of any vector bites, rodents near the houses, and history regarding vaccination status of the child were obtained. Under strict aseptic precautions, 3-5 $\mathrm{ml}$ blood was collected from children using sterile syringes.

The laboratory parameters looked at include complete hemogram, erythrocyte sedimentation rate, C-reactive protein, liver function tests, and renal function tests. For diagnosing infections, Widal test for enteric fever, peripheral smear for malarial parasites, IgM ELISA for Leptospira, IgM and NS1 antigen ELISA (Panbio) for dengue, and IgM ELISA (Panbio) for chikungunya were performed. For diagnosing scrub typhus, the serum sample was tested for Weil-Felix reaction and scrub typhus IgM ELISA using INBIOS kit. A rapid card test was done in addition to this. Antigens for Weil-Felix reaction was obtained from King Institute of Preventive Medicine and Research, Guindy. A single titer of $\geq 1: 160$ or 4 -fold rises in titer from two consecutive samples submitted a week apart is considered significant. Rapid card test by SD Bioline Tsutsugamushi, qualitative test for the detection of antibodies (IgG, IgM, and IgA) to O. tsutsugamushi was used.

The cases which were found to be positive with scrub typhus were noted. Coinfections are quite common, so if the child test's positive for either WIDAL or leptospirosis was also taken and analyzed. The cases were evaluated with their habitat and any conclusive finding was noted.

\section{RESULTS}

All the acute febrile illness of pediatric cases attending the hospital was taken into account. The current study observed about 280 cases. The age and gender analysis of the children included in this study are depicted in Table 1.

Gender-wise analysis of the study population was recorded as $62.1 \%$ and $37.9 \%$ among male and female children, respectively. Of the 20 scrub typhus positive cases, 12 were male and 8 were female.

The average duration of symptoms of scrub typhus positive cases was 6-8 days (Fig. 1); where the clinical manifestations were documented and evaluated in accordance with the severity of the disease (Table 2).

In this study, it was observed that the Weil-Felix test gave more false positive and false negative; whereas the sensitivity and specificity of Weil-Felix test were found to be $57.1 \%$ and $85.7 \%$, respectively

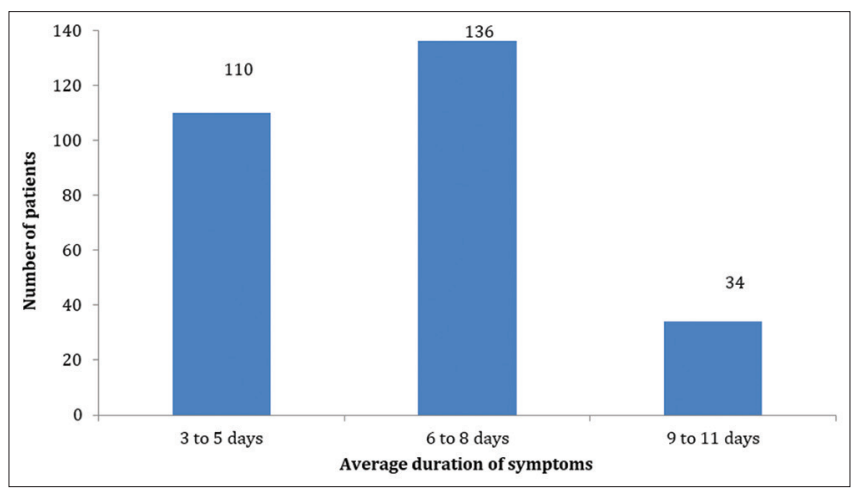

Fig. 1: Duration of symptoms in study population $(n=280)$

Table 1: Age-wise prevalence of the study population

\begin{tabular}{llll}
\hline Age (in years) & Male & Female & Number of cases $(\mathbf{n = 2 8 0})$ \\
\hline $4-6$ & 48 & 28 & $76(27.1)$ \\
$7-9$ & 76 & 34 & $110(39.3)$ \\
$10-12$ & 50 & 44 & $94(33.6)$ \\
& $174(62.1)$ & $106(37.9)$ & $280(100)$ \\
\hline
\end{tabular}

Figure in parenthesis denoted percentages
(Table 3). This implies that Weil-Felix is not a diagnostic test and further it was authenticated that the rapid card test and IgM ELISA tests gave similar results in all cases.

\section{DISCUSSION}

The prevalence of scrub typhus varies from $8 \%$ to $60 \%$ in different countries [8], and in this study, we described the prevalence and clinical profile of this illness in the pediatric patients at a tertiary hospital in South India. Our study population revealed 7.1\% (280 cases screened) positivity for scrub typhus while a study done in another part of South India reported 24\% (180 cases screened) positivity for scrub typhus [9]. The mean age group of the study population was 7-9 years $(39.3 \%)$, which is similar to a study done in Taiwan [10].

Literature states that scrub typhus is an acute febrile illness but in our study population, the duration of fever was around 8-10 days. Hence, the current study emphasizes the fact that any patient who presents with prolonged fever of more than week duration should also be tested for scrub typhus much in occurrence with another South Indian study [11].

In spite of the availability of effective antibiotic therapy, scrub typhus continues to cause significant morbidity and mortality due to lack of diagnostic facilities for the detection of the early infection phase, when antibiotic treatment is most effective [12]. Scrub typhus has to be differentiated from other febrile illnesses by Weil-Felix test, Card test, and IgM ELISA with ease.

Astudy conducted in Thailand usingSD Biolineimmunochromatographic test had reported sensitivity and specificity to be $66.7 \%$ and $98.4 \%$, respectively [12]. Among Korean population, it was reported higher sensitivity (72.6\%) of SD Bioline ICT while ImmuneMed RDT was found to be more sensitive $(98.6 \%)$ than SD Bioline RDT $(84.8 \%)[13,14]$. The retrospective diagnosis of ImmuneMed RDT exhibited $94.0 \%$ identity with ELISA using South Indian patient serum samples in this study [14]. It was found that the sensitivity of $44 \%$ and $87 \%$ was observed with Weil-Felix test and IgM ELISA, respectively [15].

In this study, $14.2 \%$ of cases were coinfected with leptospirosis, typhoid, and dengue much in concurrence with a study done in the Himalayan

Table 2: Clinical manifestations of scrub typhus positive patients $(n=20)$

\begin{tabular}{ll}
\hline Clinical symptoms & Number of cases $(\mathbf{n = 2 0})$ \\
\hline Fever & $20(100)$ \\
Nausea & $14(70)$ \\
Vomiting & $14(70)$ \\
Abdominal pain & $6(30)$ \\
Diarrhea & $2(10)$ \\
Body pain & $12(60)$ \\
Rash & $6(30)$ \\
Jaundice & $2(10)$ \\
Breathlessness & $1(5)$ \\
Cough & $10(50)$ \\
Headache & $14(70)$ \\
seizure & $1(5)$ \\
\hline
\end{tabular}

Figure in parenthesis denoted percentages

Table 3: Comparison of test results of scrub typhus positive cases $(n=20)$

\begin{tabular}{lll}
\hline Tests & $\begin{array}{l}\text { Number of } \\
\text { cases positive } \\
\text { for scrub typhus }\end{array}$ & $\begin{array}{l}\text { Number of } \\
\text { cases negative } \\
\text { for scrub typhus }\end{array}$ \\
\hline Weil-Felix test & $11(55)$ & $9(45)$ \\
Card test & $20(100)$ & - \\
IgM ELISA & $20(100)$ & - \\
\hline
\end{tabular}

Figure in parenthesis denotes percentage 
region wherein coinfections (mainly dengue fever and leptospirosis) were observed. [16].

In our study, the scrub typhus infection among pediatric cases, the mean age group affected was 7-9 years and out of the 20 positive cases, 12 were male and 8 were male and female children, respectively. According to our study, the major predisposing factor for the infection was dwelling with vegetation around their residence and the major symptoms were fever, headache, nausea, and vomiting. The sensitivity and specificity of both Card test and IgM ELISA were found to $100 \%$. Dengue, leptospirosis, and typhoid were the common coinfections observed among scrub typhus positive children.

\section{Recommendations of current study}

The study recommends that scrub typhus should be included in the differential diagnosis of fever of unknown origin in children (irrespective of its duration) along with other common communicable diseases, which are endemic in this region. This will help in the proper early diagnosis which would facilitate timely and effective treatment, thereby avoiding complications related to scrub typhus.

\section{CONCLUSION}

In India, the diagnosis of scrub typhus is difficult due to its varied symptoms in children and less availability of specific laboratory investigations (serological tests) in the rural setups. In developing countries including India, the diagnosis of scrub typhus should be based mainly on a high index of clinical suspicion coupled with appropriate laboratory correlation and epidemiological evaluation. It is prudent to recommend standardized antibiotic policy in children with febrile illness with evidence of multiorgan involvement to avoid complications due to this infection.

\section{ACKNOWLEDGEMENT}

Authors wish to acknowledge Indian Council of Medical Research (ICMR) for recognizing and providing financial support for this project under the scheme of short-term studentships (STS).

\section{AUTHORS' CONTRIBUTIONS}

Dr. A. Anupriya is a principal investigator who identified the patients and streamlined the diagnostic procedures. Mr. B. Rajkumar is a student investigator collected the samples and performed the methodology in time. Dr. N. Prabhusaran is a coinvestigator who supported the scholar to perform the laboratory works and compiled the data. Dr. S. Priya Banthavi is a coinvestigator who differentiated the PUO cases and evaluated the clinical manifestations.

\section{CONFLICTS OF INTEREST}

No.

\section{FUNDING}

This work was funded by Indian Council of Medical Research (ICMR) under the scheme of short-term studentships.

\section{REFERENCES}

1. Trowbridge P, Divya P, Samuel PK, Varghese G. The prevalence and risk factors for scrub typhus in South India. Open Forum Infect Dis 2016;3:576-82.

2. Pathak S, Chaudhary N, Dhakal P, Shakya D, Dhungel P, Neupane G, et al. Clinical profile, complications and outcome of scrub typhus in children: A hospital based observational study in central Nepal. PLoS One 2019;14:e220905.

3. Peter JV, Sudarsan TI, Prakash JA, Varghese GM. Severe scrub typhus infection: Clinical features, diagnostic challenges and management. World J Crit Care Med 2015;4:244-50.

4. Kamarasu K, Malathi M, Rajagopal V, Subramnai K, Jagadeeshramasamy D, Elizabeth M. Serological evidence for wide distribution of spotted fevers and typhus fever in Tamil Nadu. Indian J Med Res 2007:126:128-30.

5. Chakraborty S, Sarma N. Scrub typhus: An emerging threat. Indian J Dermatol 2017;62:478-85.

6. Paris DH, Shelite TR, Day NP, Walker DH. Unresolved problems related to scrub typhus: A seriously neglected life-threatening disease. Am J Trop Med Hyg 2013;89:301-7.

7. Palanivel S, Nedunchelian K, Poovazhagi V, Raghunadan R, Ramachandran P. Clinical profile of scrub typhus in children. Indian J Pediatr 2012;79:1459-62.

8. Ramyasree A, Kalawat U, Rani N, Chaudhury A. Seroprevalence of scrub typhus at a tertiary care hospital in Andhra Pradesh. Indian J Med Microbiol 2015;33:68-72.

9. Somashekar HR, Moses PD, Pavithran S, Grace ML, Agarwal I, Rolain JM, et al. Magnitude and features of scrub typhus and spotted fever in children in India. J Trop Pediatr 2006;52:228-9.

10. Chanta C, Chanta S. Clinical study of 20 children with scrub typhus at Chiang Rai Regional hospital. J Med Assoc Thail Chotmaihet Thangphaet 2005;88:1867-72.

11. Jacob S, Sekkizhar G, Kanagasabai S, Gopal P, Gopal T, Elumalai S. Seroprevalence and clinical manifestations of scrub typhus infection in Chennai city: A cross-sectional study. Int J Health Allied Sci 2018;7:201-3.

12. Silpasakorn S, Waywa D, Hoontrakul S, Suttinont C, Losuwanaluk K, Suputtamongkol Y. Performance of SD bioline tsutsugamushi assays for the diagnosis of scrub typhus in Thailand. J Med Assoc Thai 2012;95 Suppl 2:18-22.

13. Lee KD, Moon C, Oh WS, Sohn KM, Kim BN. Diagnosis of scrub typhus: Introduction of the immunochromatographic test in Korea. Korean J Intern Med 2014;29:253-5.

14. Kim YJ, Park S, Premaratna R, Selvaraj S, Park SJ, Kim S, et al. Clinical evaluation of rapid diagnostic test kit for scrub typhus with improved performance. J Korea Med Sci 2016;31:1190-6.

15. Prakash JA, Abraham OC, Mathai E. Evaluation of tests for serological diagnosis of scrub typhus. Trop Doct 2006;36:212-3.

16. Raina S, Raina RK, Agarwala N, Raina SK, Sharma R. Co-infections as an aetiology of acute undifferentiated febrile illness among adult patients in the sub-Himalayan region of North India. J Vector Borne Dis 2018;55:130-6. 